\title{
A Comparison of Hem-o-lok® and ENDOLOOP® for Ligation of Appendicular Stump in Improving Outcomes of Laparoscopic Appendicectomy
}

\author{
R. Sivamarieswaran ${ }^{1}$, Aravindan S.P2, D.S.A. Mahadevan ${ }^{3}$, T. Vidyalakshmi ${ }^{4}$ \\ 1,3 Department of General Surgery, SRM Medical College Hospital and Research Centre, SRM University, \\ Potheri, Chengalpattu, Tamil Nadu, India. ${ }^{2}$ Department of General Surgery, Nalam Hospital, Salem, Tamil \\ Nadu, India. ${ }^{4}$ Department of Plastic Surgery, SIMS Hospital, Chennai, Tamil Nadu, India.
}

\section{ABSTRACT}

\section{BACKGROUND}

Acute appendicitis is a common condition that presents as an emergency, requiring definitive surgery. The ligation of appendicular stump is one of the important steps in the laparoscopic appendicectomy. Various techniques have been used and researched for the closure of stump of appendix but the method with superior results has not been demonstrated till now. This study aims to compare the advantages and outcomes of using ENDOLOOP® and Hem-o-lok®, a non-absorbable polymer for ligating the appendicular stump.

\section{METHODS}

This was a longitudinal follow up study of 60 consecutive patients who were planned for elective laparoscopic appendicectomy, at a tertiary care institution between April 2018 and September 2019. Patients above the age of 17 years were selected for undergoing elective laparoscopic appendicectomy and grouped into two groups, group 1 - ligation of appendicular stump by Hem-o-lok® and group 2 - ligation with ENDOLOOP® by sequential allocation method.

\section{RESULTS}

Sixty patients who were selected for elective laparoscopic appendicectomy were included in the study. Thirty patients underwent ligation with Hem-o-lok $®$ clips; thirty patients underwent ligation with ENDOLOOP®. The average duration of surgery for the Hem-o-lok $₫$ was 41.33 minutes and for ENDOLOOP $®$ was 49.83 minutes ( $\mathrm{p}$ value $=0.006$ ). The average cost of ligation of appendicular stump using Hem-o-lok® was Rs. 320. The average cost in ligation of appendicular stump using ENDOLOOP® was Rs. $650(\mathrm{p}<0.001)$. The average size of the appendix ligated using the Hem-o-lok® group was $9.03 \mathrm{~mm}$ and for ENDOLOOP $® 9.23 \mathrm{~mm}(\mathrm{p}=0.13)$. There were no intra operative or post-operative complications in any of the two groups.

\section{CONCLUSIONS}

The use of Hem-o-lok $®$ in the ligation of appendicular stump has proved to be good and a safe alternative to ENDOLOOP® in laparoscopic appendicectomy. Hem-o-lok® group showed reduced operating time with less expenditure when compared to the ENDOLOOP® group.

\section{KEY WORDS}

Laparoscopic Appendicectomy, Hem-o-lok®, Endo Loop
Corresponding Author: Dr. Aravindan S.P.,

\# 257/2 Rathina Samy Puram, VIAThammannan Road, Salem-636009, Tamil Nadu, India.

E-mail:aravindansp4@gmail.com

DOI: $10.14260 /$ jemds/2021/45

How to Cite This Article:

Sivamarieswaran $R$, Aravindan SP, Mahadevan DSA, et al. A comparison of Hem-o-lok $\AA$ and ENDOLOOP $®$ for ligation of appendicular stump in improving outcomes of laparoscopic appendicectomy. J Evolution Med Dent Sci 2021;10(04):207-211, DOI: $10.14260 / \mathrm{jemds} / 2021 / 45$

Submission 18-09-2020,

Peer Review 23-11-2020,

Acceptance 30-11-2020,

Published 25-01-2021.

Copyright (@) 2021 R. Sivamarieswaran et al. This is an open access article distributed under Creative Commons Attribution License [Attribution 4.0 International (CC BY 4.0)] 


\section{BACKGROUND}

Acute appendicitis is one of the most common general surgical conditions to be treated as an emergency. Laparoscopic appendicectomy was useful in drastically reducing hospital stay, operative duration, post op complications, post op pain, with better cosmesis and early oral feeding and quick return to routine work. ${ }^{1}$ Appendicular stump closure is the most important step in laparoscopic appendicectomy. Appendicular stump closure is done to avoid major complications such as postoperative fistula formation, peritonitis and sepsis. In laparoscopic appendicectomy (LA), ligation of appendix base can be difficult because intracorporeal knotting requires good surgical skill in laparoscopy or the surgeon may not be sure of reliability of the knot. This situation has led surgeons to seek different alternatives for ligation of appendicular stump in LA.

The optimal method of closing the appendicular stump in laparoscopy should ideally be technically easy to use, safe, accessible, reliable, with less operative duration and cost effective. This is the reason for which different methods have been invented, researched and used to ligate the appendicular stump. ${ }^{2}$ Appendicular stump can be ligated by various methods during laparoscopic appendicectomy like ENDOLOOP $\AA$, metal endoclips, endo-stapler, harmonic or bipolar endocoagulation, Hem-o-lok $®$ clips and intracorporeal suture. ${ }^{3}$

In current scenario of the ever-growing expenditure in healthcare, the cost of any medical or surgical intervention is under constant evaluation. Methods to minimize the cost and to maximize care delivered to the patient are being constantly explored. Discovering various methods to achieve the similar outcomes at a lower cost is one important way to increase value of care. The major cost of any surgery is the materials and the instruments used. In field of minimal access surgery, the choice of instruments and materials chosen for any procedure depends on the operating surgeon's choice, skill of the surgeon and what instruments are available at that particular hospital. The ability to reduce costs in laparoscopy has been tried by standardizing instruments and by improving the design of instruments for an easy learning curve.

Other options include introducing less expensive instruments for achieving similar outcomes seen with an expensive operative setup. ${ }^{4}$ Hem-o-lok ${ }^{\circledR}$ is a polymer clip which is being used in various laparoscopic surgeries like laparoscopic cholecystectomy, nephrectomy and other vascular procedures. This study aims in comparing the advantages and limitations of the Hem-o-lok ${ }^{\circledR}$ clips versus ENDOLOOP® sutures for the ligation of appendix stump in laparoscopic appendicectomy. The advantages and limitations of Hem-o-lok $₫$ versus ENDOLOOP $®$ are compared by using parameters like cost of surgery, operating time, intra operative complications and post-operative complications.

\section{METHODS}

This study was a longitudinal follow up study of patients who underwent elective laparoscopic appendicectomy in SRM Medical College Hospital and Research Centre, Tamilnadu, a tertiary care centre. This study commenced in April 2018, after ethical approval from the human ethics committee of the institute and ended in September 2019. All patients above the age of 18 years who were planned to undergo elective laparoscopic appendicectomy were included in this study. Patients not giving informed consent for the study, all patients with symptoms, signs and radiological investigations suggestive of appendicular perforation, abscess, local peritonitis and mass formation and patients who had a probability of laparoscopic appendicectomy converting to open appendicectomy were excluded from the study.

A written informed consent was obtained from all patients before the procedure. Sample size was calculated with a previous original study done by Samir Delibegovic in 2009. The study showed a significant difference in operating time between the two groups. Operating time of ENDOLOOP $®$ and Hem-o-lock was 47.1 $\pm 6.7 \mathrm{~min}$ and $38.7 \pm 5.0 \mathrm{~min}$ with $\alpha=0.01$ and $\beta=0.1$ with $p$ value $<0.05$. The minimum sample size was estimated to be 25 in each group. Assuming $20 \%$ refusal rate the sample size increased to 30 in each group.

Patients who presented with a confirmed diagnosis of acute appendicitis were initially managed conservatively with antibiotics and were posted for the following day for elective laparoscopic appendicectomy. Routine tests included complete haemogram, urine analysis and microscopy, serum electrolytes, renal function tests, to help ascertain the diagnosis of acute appendicitis. Ultrasonography of the abdomen was done for these patients to confirm the diagnosis of appendicitis. Routine chest X-ray was also done for the purpose of pre anaesthetic clearance.

The patients were divided into two groups and sampling was based on sequential allocation. One group of 30 patients underwent closure of appendicular stump with Hem-o-lok $₫$ clips and another group of 30 patients underwent closure of appendicular stump with ENDOLOOP® ligatures.

\section{Laparoscopic Appendicectomy with Hem-o- lok ${ }^{\circledR}$ clips}

After administering general anaesthesia (GA), patient was laid in supine position, under strict aseptic precautions, parts were painted and draped. A $10 \mathrm{~mm}$ optical port was inserted trans umbilical or via infra umbilical incision. A $5 \mathrm{~mm}$ trocar was inserted at the LIF and $5 \mathrm{~mm}$ trocar inserted in the supra pubic areas following the law of triangulation. Appendix is identified and the meso-appendicular window is created. The appendicular artery is cauterised and the base of the appendix is isolated. The camera is changed to the $5 \mathrm{~mm}$ scope and inserted through the LIF port. The base of the appendix is ligated with Hem-O- Lok clips using the Hem-o-lok® applicator through the $10 \mathrm{~mm}$ port. Another clip is inserted and placed above the base of appendix. The appendicular base is transacted between the two clips and the specimen is delivered through the $10 \mathrm{~mm}$ trocar. 1 - 0 Vicryl sutures are used for closing the sheath and the skin closure of all 3 port sites are done by using 3 - 0 Ethilon sutures.

Laparoscopic Appendicectomy with ENDOLOOP ${ }^{\circledR}$ After administering GA, patient was laid in supine position, under strict aseptic precautions, parts were painted and draped. A $10 \mathrm{~mm}$ trans umbilical or infra umbilical incision is made, and a $10 \mathrm{~mm}$ trocar is inserted for the scope and the appendix is visualised. A $5 \mathrm{~mm}$ trocar is inserted at the LIF and 
supra pubic areas following the law of triangulation and the meso-appendicular window is created. Cauterisation of the appendicular artery is done, and the appendicular base is ligated with an ENDOLOOP $\AA$. The next ENDOLOOP $\AA$ is applied above the previous knot and the appendicular base is transacted between the two knots and delivered through the $10 \mathrm{~mm}$ trocar. 1 - 0 Vicryl sutures are used for closing the sheath and the skin closure at all 3 port sites is done using 2 Ethilon sutures.

The procurement of the ENDOLOOP $\AA$ and Hem-o-lok $\AA$ clips was from the hospital pharmacy. Each Hem-o-lok $₫$ cartridge contains 6 clips. Average of around 2 clips were used for each patient. The remaining clips were sterilised using ethylene oxide (EO) and used again. Depending on the number of clips used the patients were charged from the operating theatre.

\section{Statistical Analysis}

Data entry was done in Excel and analysis done using SPSS software. Descriptive statistics calculated as percentages. Association between categorical variables were tested for significance by chi square test. Association between quantitative variables and type of surgical methods were tested for statistical significance by unpaired $t$ test. $\mathrm{P}$ value < 0.05 was considered statistically significant.

\section{RESULTS}

Total number of patients who underwent laparoscopic appendicectomy were 60 , among whom 30 patients were belonging to Group A and 30 patients were of Group B. Patients were allocated based on sequential allocation. Group A (Hem-o-lok®) had 30 patients. Group B (ENDOLOOP $®)$ had 30 patients.

\section{Distribution by Age and Gender}

The age distribution of patients among both the groups was found to be similar. In the Hem-o-lok® group, $43.3 \%$ of patients were in the range of $26-35$ years. In the ENDOLOOP $®$ group $40.0 \%$ of patients were in the similar age range. Majority of the patients were male in both the groups. Total number of males in Hem-o-lok@ group were $53.3 \%$ and ENDOLOOP $®$ were $56.7 \%$. Total number of females in the Hem-o-lok® group were $46.7 \%$ and ENDOLOOP $®$ group were $43.3 \%$. (Table 1 )

\begin{tabular}{|ccccccc|}
\hline Variable & \multicolumn{2}{c}{ Hem-o-lok ${ }^{\circledR}$} & \multicolumn{2}{c}{ ENDOLOOP® } & \multicolumn{2}{c|}{ Total } \\
Number & $\%$ & Number & $\%$ & Number & $\%$ \\
Age in years & & & & & & \\
16 to 25 & 8 & $26.7 \%$ & 7 & $23.3 \%$ & 15 & $25.0 \%$ \\
Above 55 & 1 & $3.3 \%$ & 1 & $3.3 \%$ & 2 & $3.3 \%$ \\
Gender & & & & & & \\
Male & 16 & $53.3 \%$ & 17 & $56.7 \%$ & 33 & $55 \%$ \\
Female & 14 & $46.7 \%$ & 13 & $43.3 \%$ & 27 & $45 \%$ \\
TOTAL & 30 & 100.0 & 30 & 100.0 & 60 & 100.0 \\
\hline \multicolumn{7}{|c|}{ Table 1. Demographic Distribution of the Study Participants } \\
\hline
\end{tabular}

\section{Distribution by Duration of Surgery}

In the Hem-o-lok@ group majority of patients (56.7\%) were operated less than $45 \mathrm{~min} .36 .7 \%$ were operated from $45 \mathrm{~min}$ to one hour and $6.7 \%$ took more than one hour. While in the ENDOLOOP $®$ group majority of patients $(76.7 \%)$ were operated in more than $45 \mathrm{~min}$ and within one hour $18.3 \%$ were operated in less than $45 \mathrm{~min}$, while $10 \%$ were operated in more than one hour. (Table 2)

\begin{tabular}{|ccccccc|}
\hline Duration of Surgery & \multicolumn{2}{c}{ Hem-o-lok ${ }^{\circledR}$} & \multicolumn{2}{c|}{ ENDOLOOP } & \multicolumn{3}{c|}{ Total } \\
\hline & Number & $\%$ & Number & $\%$ & Number & $\%$ \\
$<45 \mathrm{Min}$ & 17 & $56.7 \%$ & 4 & $13.3 \%$ & 21 & $35.0 \%$ \\
$>45 \mathrm{~min}-$ & 11 & $36.7 \%$ & 23 & $76.7 \%$ & 34 & $56.7 \%$ \\
$<1 \mathrm{hr}$. & 2 & $6.7 \%$ & 3 & $10.0 \%$ & 5 & $8.3 \%$ \\
$>1 \mathrm{hr}$. & 30 & $100 \%$ & 30 & $100 \%$ & 60 & $100 \%$ \\
\hline Total & Table 2. Duration of Surgery \\
\hline
\end{tabular}

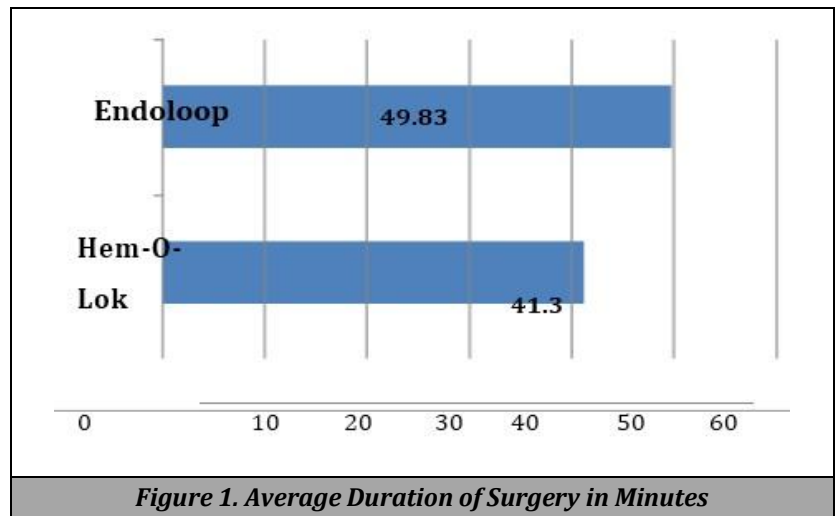

The average duration of surgery for the Hem-o-lok® is $41.3 \mathrm{~min}(\mathrm{SD}=12.3 \mathrm{mins}$ ) and for ENDOLOOP $\AA$ is $49.83 \mathrm{mins}$ ( $\mathrm{SD}=10.5 \mathrm{mins}$ ). The difference in duration of surgery was statistically significant $(\mathrm{P}$ value $=0.006)$.

\begin{tabular}{|c|c|c|c|c|}
\hline Quantity & One & Two & Three & $\begin{array}{l}\text { Average Number } \\
\text { Used per Patient }\end{array}$ \\
\hline Hem-o-lok® & $\mathrm{Nil}$ & 28 & 2 & 2.06 \\
\hline ENDOLOOP® & 30 & Nil & Nil & 1 \\
\hline \multicolumn{5}{|c|}{$\begin{array}{l}\text { Table 3. Number of Hem-o-lok® Versus ENDOLOOP® } \\
\text { Used for Ligation of Appendicular Stump }\end{array}$} \\
\hline
\end{tabular}

\section{Cost of Closure of Appendicular Stump}

The average cost of ligation of appendicular stump was calculated with mean and t test. The average cost of ligation of appendicular stump using Hem-o-lok® was Rs 320 (SD = 76 Rs). The average cost in ligation of appendicular stump using ENDOLOOP ${ }^{\circledR}$ was Rs $650(S D=0)$. The $p$ value was less than 0.001 and proved to be significant.

\section{Average Size of Appendix}

The average size of the appendix ligated using the Hem-o-lok ${ }^{\circledR}$ group was $9.03 \mathrm{~mm}$ (SD $=2.1 \mathrm{~mm}$ ). The average size of appendix ligated using the ENDOLOOP ${ }^{\circledR}$ was $9.23 \mathrm{~mm}(\mathrm{SD}=$ $1.7 \mathrm{~mm}$ ). The $\mathrm{p}$ value for both groups was 0.427 which was not significant. There were no Intra and post-operative complications for both the groups. Patients were followed up for 8 weeks and none of the patients developed any postoperative complications during follow up. 


\section{DISCUSSION}

Acute appendicitis is one of the most common general surgical conditions to be treated as an emergency. Since the invention of laparoscopic surgery, surgeons have constantly worked towards improving the methods of diagnosis, forming various scoring systems, discovering various surgical techniques to reduce cost and help reducing duration of hospital stay for the treatment of acute appendicitis. Currently, many surgeons use either ENDOLOOP®S or endostaplers to ligate the appendicular stump. A number of studies have been done to show advantages and disadvantages in both of these products. Both ENDOLOOP $囚$ s and endostaplers are safe for the closure of the appendix stump laparoscopically, but endostaplers are highly expensive and for the use of ENDOLOOP®, the surgeon requires good surgical skills and previous experience in laparoscopy. Hem-o-lok ${ }^{\circledR}$ could be used in ligation of appendicular stump, which would reduce the cost, have an easy learning curve and decrease the duration of surgery. In this study advantages and outcomes in both methods were studied.

In this study, majority of the patients were in the age group of $26-35$; both the groups had no significant difference with respect to the distribution of age. Total females in the Hem-olok® group were $46.7 \%$ and ENDOLOOP $®$ group were 43.3 $\%$. Both the groups had equal distribution. No significant value in the distribution of patients. ${ }^{5}$

Hem-o-lok ${ }^{\circledR}$ clips are available in various sizes (small, medium, large, and extra-large). Our study was done with large size Hem-o-lok $®$ clips. The study done by Chang Sik showed the mean diameter of the appendix in the Hem-o-lok $\AA$ group to be $9.9 \mathrm{~mm}$ and $9.7 \mathrm{~mm}$ in the ENDOLOOP® group. 6 So the large size of Hem-o-lok® which has a range of $5 \mathrm{~mm}$ to $13 \mathrm{~mm}$ was used in the study. Similar results were observed in our study. Our study showed an average size of $9.03 \mathrm{~mm}$ in Hem-o-lok® group and $9.23 \mathrm{~mm}$ in the ENDOLOOP® group. Delibegovic studied the average size of appendix. The average size of the base of appendix in the phlegmon form was $10.26 \pm$ 3.18 , in the gangrene form $12.48 \pm 3.55$, and in the perforated form $12.47 \pm 3.68$. The perforated form had the maximum size of base which was $23.13 \mathrm{~mm} .^{7}$

The duration of surgery was calculated between two groups. In the Hem-o-lok ${ }^{\circledR}$ group, majority of patients $(56.7$ $\%$ ) were operated less than $45 \mathrm{~min} .36 .7 \%$ were operated from 45 min to one hour and $6.7 \%$ took more than one hour. While in the ENDOLOOP $®$ group, majority of patients (76.7\%) were operated more than $45 \mathrm{~min}$ and within one hour. $13.3 \%$ were operated less than $45 \mathrm{~min}$, while $10 \%$ were operated more than one hour. The Average duration of surgery for the Hem-o-lok $®$ is $41.33 \mathrm{~min}$ and for ENDOLOOP $®$ is $49.83 \mathrm{~min}$. $\mathrm{P}$ value was 0.006 and proved to be significant $(<0.05)$. Study done by Elif Colak showed that the operating time in the Hemo-lok $\AA$ group was $64.7 \mathrm{~min}$ and ENDOLOOP $®$ showed 75.4 min which shows less operating time in Hem-o-lok $®$ group but $p$ value was not significant ( $p$ 0.072). ${ }^{8}$ Samir Delibegovic et al showed that the operating time in the Hem-o-lok® group was $47.1 \mathrm{~min}$ and ENDOLOOP $®$ was $38.7 \mathrm{~min}$ which showed less operating time in Hem-o-lok® group and a significant $\mathrm{p}$ value (p 0.00001). ${ }^{9}$

Only a single clip was applied at the base of the appendix and one clip with the specimen. The average numbers of clips used were 2.06. Study done by Lars Ivo Partecke et al showed that LA using a single Hem-o-lok $®$ is safe and sufficient rather than multiple clips which would increase the cost. Both our study and other studies showed that single clip is safe and an efficient method for ligating the base of inflamed appendix. ${ }^{10}$

The average cost for the ligation of appendicular stump using Hem-o-lok® was Rs: 320 . The average cost in ligation of appendicular stump using ENDOLOOP ${ }^{\circledR}$ was Rs: 650 . This proved to be statistically significant for the Hem-o-lok® group $(p=0.001)$. Study done by Elif Colak showed that the cost of surgery in the Hem-o-lok® group was $\$ 30$ and ENDOLOOP $®$ showed $\$ 120$ which shows less costs in Hem-o-lok® group. ${ }^{8}$ Similarly, Chang Sik et al showed similar results with less costs in the Hem-o-lok ${ }^{\circledR}$ group when compared to ENDOLOOP ${ }^{\circledR}{ }^{6}$

None of the patients in our study had any post-operative complications like appendicular abscess, cutting through of the appendicular base or post op pain. Study done by Christopher Soll et al proved that ligating the appendicular stump by the non-absorbable Hem-o-lok® clips resulted in lesser incidence of intra-abdominal surgical abscesses, when compared to the ones where ligation was done by applying ENDOLOOP $® .11$ Two studies done by Samir Delibegovic et al in 2009 and 2012 had no intraoperative or post op complications which were similar to our study. ${ }^{9,12}$

Study done by Samir Delibegovic compared the tissue reaction between ENDOLOOP $®$, Hem-o-lok® clips. The study showed only some milder postoperative inflammatory changes were observed in the stapler group, than by the Hemo-lok $\AA^{\circledR}$ group. However, comparing the price of the Hem-olok $₫$ clip and the easy application of clip, its use is still favored during laparoscopic appendicectomy and lesser complications were noted with ENDOLOOP ${ }^{13,14}$

Safety of Hem-o-lok $\AA$ and ENDOLOOP $®$ to ligate the appendicular base in laparoscopic appendicectomy was analysed in multiple studies. Caroloine Et al proved that Hemo-lok® clips are safe alternatives for ligation of the appendicular base in laparoscopic appendicectomy. They are cost effective without any evidence of increased complications. Ali Aminian et al also showed that "Hem-olok® can be used safely in various laparoscopic procedures when the proper technique is learned". 15 In our study, out of the 30 patients who underwent laparoscopic appendicectomy none of the patients developed any intraoperative postoperative complications which shows that Hem-o-lok ${ }^{\circledR}$ clips are safe and can be used to ligate the appendicular stump.

\section{CONCLUSIONS}

In a developing country like India, resources for advanced laparoscopic training are scarce. Hence, techniques with an easy learning curve should be incorporated in different advanced laparoscopic procedures. We propose that Hem-olok® will be an easier technique to master by the beginners thus reducing the operating time. Cost comparison analysis has statistically proved that Hem-o-lok® is cheaper than ENDOLOOP®. The average duration of surgery was less with Hem-o-lok $®$ when compared to Endo loop. Patients in the Hem-o-lok® group had no intra operative or post-operative complications. Hence, Hem-o-lok $\AA$ is a safe method for the ligation of appendicular stump. 
Data sharing statement provided by the authors is available with the full text of this article at jemds.com.

Financial or other competing interests: None.

Disclosure forms provided by the authors are available with the full text of this article at jemds.com.

\section{REFERENCES}

[1] Meljnikov I, Radojcic B, Grebeldinger S, et al. History of surgical treatment of appendicitis . Med Pregl 2009;62(910):489-92.

[2] Mayir B, Ensari CO, Bilecik T, et al. Methods for closure of appendix stump during laparoscopic appendicectomy procedure. Ulus Cerrahi Derg 2015;31(4):229-31.

[3] Gomes CA, Junior CS, de Peixoto RO, et al. Appendiceal stump closure by metal endoclip in the management of complicated acute appendicitis. World J Emerge Surg 2013;8(1):35.

[4] Reinke CE, Tabone LE, Fong P, et al. Safety and efficacy of polymeric clips for appendicealstump closure. JSLS 2016;20(3):e2016.00045.

[5] Bozkurt MA, Ünsal MG, Kapan S, et al. Two different methods for appendiceal stump closure: metal clip and Hem-o-lok® clip. J Laparoendosc Adva Surg Tech A 2014;24(8):571-3.

[6] Hue CS, Kim JS, Kim KH, et al. The usefulness and safety of Hem-o-lok® clips for the closure of appendicular stump during laparoscopic appendicectomy. Journal of the Korean Surgical Society 2013;84(1):27-32.

[7] Delibegović S, Mehmedović Z. The influence of the appendiceal base diameter on appendix stump closure in laparoscopic appendicectomy. World J Surg 2016;40(10):2342-7.
[8] Colak E, Kement M, Ozlem N, et al. A comparison of nonabsorbable polymeric clips and ENDOLOOP ${ }^{\circledR}$ ligatures for the closure of the appendicular stump in laparoscopic appendicectomy: a prospective, randomized study. Surg Laparosc Endosc Percutan Tech 2013;23(3):255-8.

[9] Delibegović S, Matović E. Hem-o-lok® plastic clips in securing of the base of the appendix during laparoscopic appendicectomy. Surgi Endosc 2009;23(12):2851-4.

[10] Partecke LI, Kessler W, von Bernstorff W, et al. Laparoscopic appendicectomy using a single polymeric clip to close the appendicular stump. Langenbecks Arch Surg 2010;395(8):1077-82.

[11] Soll C, Wyss P, Gelpke H, et al. Appendiceal stump closure using polymeric clips reduces intra-abdominal abscesses. Langenbecks Arch Surg 2016;401(5):661-6.

[12] Delibegović $S$. The use of a single Hem-o-lok® clip in securing the base of the appendix during laparoscopic appendicectomy. J Laparoendosc Adv Surg Tech A 2012;22(1):85-7.

[13] Delibegović S, Katica M, Latić F, et al. Biocompatibility and adhes ion formation of different ENDOLOOP $®$ ligatures in securing the base of the appendix. JSLS 2003;17(4):5438.

[14] Delibegović S, Iljazović E, Katica M, et al. Tissue reaction to absorbable ENDOLOOP $®$, nonabsorbable titanium staples and polymer Hem-o-lok® clip after laparoscopic appendicectomy. JSLS 2011;15(1):70-6.

[15] Aminian A, Khorgami Z. Hem-o-lok ${ }^{\circledR}$ clip is safe in minimally invasive general surgery: a single center experience and review of data from food and drug administration. J Minim Invasive Surg Sci 2012;1(2):52-7. 